\title{
An integrative framework of coexistence mechanisms in competitive metacommunities
}

\author{
Bertrand Fournier, Nicolas Mouquet, Mathew A. Leibold and Dominique Gravel
}

B. Fournier(bfourni@gmail.com) and N. Mouquet, UMR 5554 ISEM, CNRS-IRD-UM, Univ. de Montpellier, Montpellier, France. NM also at: UMR 9190 MARBEC, CNRS-IRD-IFREMER-UM, Univ. de Montpellier, Montpellier, France. - M. A. Leibold, Dept of Integrative Biology, Univ. of Texas at Austin, Austin, TX, USA. - D. Gravel, Dépt de biologie, Univ. de Sherbrooke, Sherbrooke, QC, Canada.

\begin{abstract}
Species distribution in a metacommunity varies according to their traits, the distribution of environmental conditions and connectivity among localities. These ingredients contribute to coexistence across spatial scales via species sorting, patch dynamics, mass effects and neutral dynamics. These mechanisms however seldom act in isolation and the impact of landscape configuration on their relative importance remains poorly understood. We present a new model of metacommunity dynamics that simultaneously considers these four possible mechanisms over spatially explicit landscapes and propose a statistical approach to partition their contribution to species distribution. We find that landscape configuration can induce dispersal limitations that have negative consequences for local species richness. This result was more pronounced with neutral dynamics and mass effect than with species sorting or patch dynamics. We also find that the relative importance of the four mechanisms varies not only among landscape configurations, but also among species, with some species being mostly constrained by dispersal and/or drift and others by sorting. Changes in landscape properties might lead to a shift in coexistence mechanisms and, by extension, to a change in community composition. This confirms the importance of considering landscape properties for conservation and management. Our results illustrate the idea that ecological communities are the results of multiple mechanisms acting at the same time and complete our understanding of spatial processes in competitive metacommunities.
\end{abstract}

A metacommunity is defined as a set of local communities linked by dispersal where species coexistence depends on niche differentiation, competitive hierarchies, drift and dispersal (Leibold et al. 2004). Metacommunity theory distinguishes four idealized coexistence mechanisms: species sorting, patch dynamics, mass effect and neutral dynamics (Leibold et al. 2004). The relative importance of niche differentiation, competitive hierarchies, drift and dispersal varies among mechanisms according to the capacity of species to disperse, experience their environment and interact with other individuals (Livingston et al. 2012). It can also change with landscape connectivity and with the distribution of environmental conditions (Biswas and Wagner 2012). Sorting of individuals by environmental conditions is a key mechanism for the mass effect and species sorting mechanisms, as species coexistence is obtained when there is a spatial variability in the competitive hierarchy among species. These two mechanisms differ because in mass effects excess of dispersal can counteract the effect of environmental sorting. With patch dynamics, coexistence is obtained through a trade-off between the competitive and colonization abilities of species. Finally, neutral dynamics consider that all species have equal competitive abilities throughout the landscape and that coexistence depends on ecological drift: an equilibrium between immigration and extinctions due to random changes in species abundances (Hubbell 2001). While not originally considered by Leibold et al (2004), different combinations of the four basic mechanisms are also likely to be important in structuring communities. In addition, dispersal limitations, where an inferior competitor successfully recruits into patches where it is not the dominant because the superior competitor is absent from the pool of propagules, can also be an important process for species coexistence (Hurtt and Pacala 1995, Hubbell et al. 1999). All together these processes determine which species will coexist at both local and regional scales and where they are found (Leibold et al. 2004, Mouquet et al. 2005, Vellend 2010). Their importance has been widely documented in both empirical and theoretical studies (Livingston et al. 2012), but it is only recently that major advances have been made toward a synthesis (Gravel et al. 2006, Leibold 2011). The metacommunity theory explicitly accounts for niche differentiation, competitive hierarchies, drift and dispersal. It is therefore a useful framework to integrate these processes and advance our understanding of coexistence and distribution across spatial scales.

A growing body of evidence suggests that different mechanisms can operate simultaneously in a metacommunity (Amarasekare et al. 2004, Mouquet et al. 2005, Gravel et al. 2006, Leibold and McPeek 2006). Leibold 
(2011) and Logue et al. (2011) proposed that species sorting, patch dynamics, mass effect and neutral dynamics can be placed within a common framework where the mechanisms are separated along three continuous axes describing ecological equivalence among species, environmental heterogeneity and dispersal. For example, altering dispersal limitations and the equivalence among species can induce a switch from neutral to species sorting (Gravel et al. 2006). Dispersal can also impact local species co-existence through a mass effect (Mouquet and Loreau 2002). The mass effect requires sufficient propagule pressure to dislodge superior competitors, which is more likely to be found in landscapes with very sharp transitions between environmental conditions. Interactions between mechanisms can also have complex consequences. Amarasekare et al. (2004) used a model coupling source-sink dynamics and life-history trade-offs to demonstrate that spatial heterogeneity can modify life-history trade-offs reducing the potential for species coexistence. In general, a change in the connectivity among patches, the frequency distribution of environmental conditions, or their spatial distribution, can impact the relative importance of niche differentiation, competitive hierarchies, drift and dispersal and, thereby, the importance of the four paradigms. For instance, it was shown with experiments that dispersal constraints imposed by particular landscape topologies such as dendritic river networks have direct consequences for metacommunity diversity and composition (Carrara et al. 2012). Recent studies modeling complex landscape structures as networks revealed that landscape topology can impact neutral metacommunity biodiversity (Economo and Keitt 2008, 2010,
Urban et al. 2009, Gravel et al. 2014), but less is known about how this can alter other processes.

Here, we introduce a general metacommunity model that integrates niche differentiation, competitive hierarchies, drift and dispersal in a spatially explicit landscape within a single framework. The model simulates competition for space in continuous landscapes (network of patches) with different spatial structure and distribution of environmental conditions. We also consider sub-models that follow the assumptions of each of the four coexistence mechanisms from metacommunity theory. These sub-models provide predictions specific to each of them against which observed species distributions could be compared. We use the sub-models to disentangle the contribution of each mechanism to species distributions. Our analysis provides important insights about which aspects of landscape heterogeneity promotes coexistence and, by extension, which species are expected to persist. We present simulations with one, two and twenty species to illustrate how landscape topology and coexistence mechanisms can influence biodiversity. We conclude by discussing the potential applications of our approach.

\section{Methods}

\section{Model description}

The model represents competition for patches in a spatially explicit landscape (Fig. 1). Each patch could be empty or host a single species. The formalism should be seen as an approximation to dominance by a single species. It has also (a)

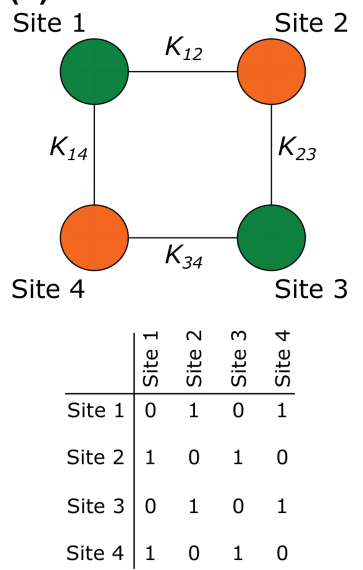

(b)
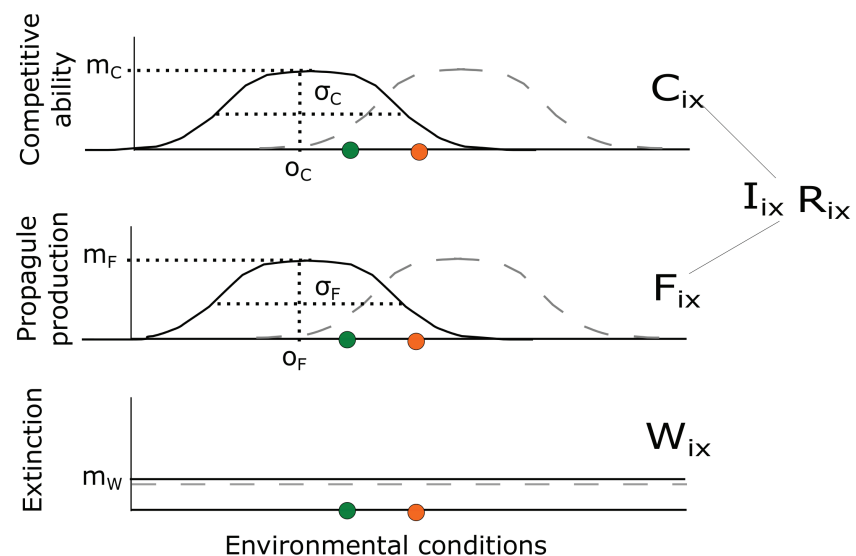

(c)
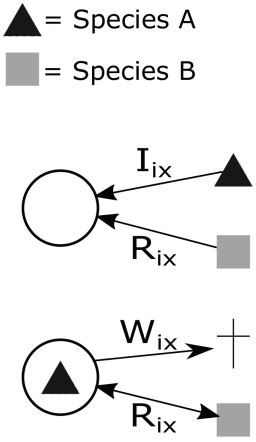

Figure 1. Simplified description of the model. (a) Hypothetical landscape composed by four patches and including environmental heterogeneity. $K_{i j}$ is a dispersal kernel based on the distance among sites. Two patches are considered connected when $K_{i j}$ is lower than a threshold Euclidean distance of $r$. The lower panel shows the connectivity among sites as an adjacency matrix where 1 indicates connected sites and 0 un-connected ones. (b) Competitive ability $\left(C_{i x}\right)$, propagule production $\left(F_{i x}\right)$ and extinction probability $\left(W_{i x}\right)$ as a function of environmental conditions for species A (solid black line) and species B (dashed grey line). Each of $C_{i x}, F_{i x}$ and $W_{i x}$ is defined by three species specific parameters: species maximal performance $(\mathrm{m})$, optimum condition $(\mathrm{o})$ and niche breadth $(\sigma)$. In our example, species A and B have equal maximal performance and niche breadth for competitive ability and propagule production, but have different environmental optima. As a result, species A is a better competitor in sites 1 and 3 (green dot) whereas species B outcompetes species A in sites 2 and 4 (orange dot). Extinction probability is constant throughout the landscape $\left(\sigma_{\mathrm{w}}=\infty\right)$ and equal among the two species. (c) Local dynamic in the case of an empty site and in the case of a site already occupied by species A. The probability that species A can colonize an empty site depends on its recruitment probability $\left(I_{i x}\right)$ and the probability that a superior competitor colonizes the empty patch $\left(R_{i x}\right)$. When species A is present at a site, it can die depending on its extinction probability $\left(W_{i x}\right)$ leaving the site empty or be replaced by a superior competitor (as a function of $R_{i x}$ ). 
been applied at the individual level (Tilman 1994), where each patch corresponds to a microsite. We represent the landscape as a spatial network where patches are nodes and connections by dispersal are vertices. The landscape consists of $\mathrm{N}$ patches $x$ of varying areas $\left(A_{x}\right)$ and environmental conditions $\left(e_{x}\right)$, which are submitted to random disturbances $\left(d_{x}\right)$. There are $S$ species $i$ in the regional species pool. Recruitment in empty patches happens as a weighted lottery among propagules reaching them. Recruitment can also happen with the replacement, by a weighted lottery again, by a superior competitor. Propagule production $\left(F_{i x}\right)$ and competitive ability $\left(C_{i x}\right)$ depend on the environmental condition of the patch.

We start from Levins' metapopulation model (Levins 1969, Levins and Culver 1971) and include elements of more recent models of metacommunity dynamics (Mouquet and Loreau 2002, Calcagno et al. 2006, Economo and Keitt 2010). The model describes the variation through time of occurrence probability (occupancy), $p_{i x}$ for each species $i$ in each patch $\mathrm{x}$, with a set of equations of the form:

$$
\begin{aligned}
\frac{d p_{i x}}{d t}= & I_{i x}\left(1-\sum_{j=1}^{S} p_{j x}-R_{i x}\right) \\
& -\frac{W_{i x} p_{i x}}{A_{x}}+\gamma R_{i x} I_{i x} \sum_{j \in \epsilon_{i x}>C_{j x}} p_{j x}-\gamma R_{j x} p_{i x}
\end{aligned}
$$

There are four terms in this equation, describing respectively from the left to the right the probability of recruitment in empty patches, the probability of local extinctions (because of stochastic dynamics and natural disturbances), the probability of colonization of patches occupied by inferior competitors and the probability of displacement by superior competitors. Note that, for all species, $i \neq j$.

The 'recruitment probability in empty patches' $I_{i x}$ is the probability that at least one propagule of species $i$ colonizes the patch $x$ if empty (with probability $1-\sum_{j=1}^{S} p_{j x}$ ):

$I_{i x}=1-\prod_{y}^{N}\left(1-C_{i x} F_{i y} K_{x y} p_{i y} A_{y}\right)$

where $C_{i x}$ is the competitive ability of offspring reaching the patch $x, F_{i y}$ is the propagule production in the patch $y$ and $K_{x y}$ the probability of a dispersal event to occur from patch $y$ to patch $\mathrm{x}$. The amount of propagules dispersed is directly proportional to the area of the patch. We specify an exponential dispersal kernel based on the pairwise Euclidean distance among patches $\left(d_{x y}\right)$ :

$K_{x y}=\exp \left(-0.5 d_{x y}\right)$

For simulations, we truncated the kernel at a threshold distance $r$ (i.e. all values above this threshold were set to zero) to limit computation time. This approach allowed us to transform $K_{x y}$ into an adjacency matrix and thereby to account for the structures of the landscape. However, this formulation does not allow for long distance dispersal. This assumption can be relaxed by setting all values above the distance threshold to a low probability.

Colonization of an empty patch requires successful establishment given local environmental conditions (Eq. 2) and the ability to exclude other species. The probability that a superior competitor colonizes the empty patch $\left(R_{i x}\right)$ is given by:
$R_{i x}=1-\prod_{j \in C_{j x}>C_{i x}}^{S}\left(1-I_{j x}\right)$

Competitive hierarchy is defined by species competitive ability in local environmental conditions $C_{i x}$ (Table 1). In other words, the species with the highest competitive ability in the colonized patch is the best competitor. But, species with lower $C_{i x}$ can still win the competition for a target patch if they have a sufficiently high production of propagules in source patches $\left(F_{i x}\right)$. Furthermore, the model allows for 'winning by forfeit' (Hurtt and Pacala 1995) because dispersal limitations can potentially prevent the most competitive species from reaching a suitable patch.

Local extinction from either demographic stochasticity or disturbance occurs with probability $W_{i x}$. The probability is species and patch specific. In the same environmental conditions, larger patches have lower extinction probability than smaller ones based on the assumption that extinction probability is inversely related to population size (Hanski 1994).

Colonization of patches occupied by inferior competitors is accounted by the third term of Eq. (1). It represents the probability that a superior competitor species excludes an established inferior competitor from a patch.

Displacement from patches by superior competitors is described in the fourth term. It represents the probability that a superior competitor colonizes the patch occupied by species $i$ and then excludes it. The parameter $\gamma$ sets the strength of displacement (Calcagno et al. 2006). Pure pre-emptive competition occurs when $\gamma=0$.

Reproductive and competitive abilities are related to the local environmental condition $\left(e_{x}\right)$ and species capacity to resist extinctions depends on disturbance intensity $\left(W_{x}\right)$. The model is general enough to account for any function relating propagule production, competitive ability and extinction probability to the environment. We consider here Gaussian functions with three parameters ( $m, o$, and $\sigma)$ to represent species competitive ability and offspring production as a function of the local environmental conditions $\left(e_{x}\right)$ and species resistance to disturbance as a function of local disturbance intensity $\left(w_{x}\right)$. Parameters and functions are explained at Table 1.

\section{Landscape construction}

Simulations are run on landscapes of various topologies (Fig. 2). We distribute $\mathrm{N}$ patches in a geographic space with $\mathrm{X}$ and $\mathrm{Y}$ coordinates in the range $[1,1000]$. Aggregation of patches is obtained by dividing the landscapes into 100 $10 \times 10$ cells, selecting a number of cells based on patch aggregation and randomly distributing the patches within them. The parameter aggr defines the number of patches within a selected cell and can vary from 1 (minimal aggregation) to $N$ (maximal aggregation where all patches are placed within the same cell). The number of cells is given by dividing the number of patches by aggr and rounding up the result. For example, distributing 100 patches with aggr $=2$ requires 50 cells. Two patches are considered connected if they are located within the same threshold Euclidean distance of $r$ previously used to truncate the dispersal kernel 
Table 1. Species parameters and associated Gaussian functions defining species performances in local conditions. The parameters $e_{x}$ and $w_{x}$ define local environmental conditions and local disturbance intensity, respectively.

\begin{tabular}{|c|c|c|c|}
\hline Parameter & Definition & Gaussian function & Definition \\
\hline $\mathrm{m}_{\mathrm{C}}$ & $\begin{array}{l}\text { Maximal competitive ability in local environmental } \\
\text { conditions }\end{array}$ & \multirow{3}{*}{$C_{i x}=m_{c i} \exp \left(-\left(\frac{\left(e_{x}-o_{C i}\right)^{2}}{2 \sigma_{C i}^{2}}\right)\right)$} & \multirow[t]{3}{*}{$\begin{array}{l}\text { Competitive ability in local } \\
\text { environmental conditions }\end{array}$} \\
\hline $\mathrm{o}_{\mathrm{C}}$ & $\begin{array}{l}\text { Environmental conditions where competitive ability is } \\
\text { maximal }\end{array}$ & & \\
\hline$\sigma_{\mathrm{C}}$ & $\begin{array}{l}\text { Competitive ability in sub-optimal environmental } \\
\text { conditions (competitive niche breadth) }\end{array}$ & & \\
\hline $\mathrm{m}_{\mathrm{F}}$ & Maximal propagule production & \multirow{3}{*}{$p\left(-\left(\frac{\left(e_{x}-o_{F i}\right)^{2}}{2 \sigma_{F i}^{2}}\right)\right)$} & \multirow{3}{*}{$\begin{array}{l}\text { Propagule production in } \\
\text { local environmental } \\
\text { conditions }\end{array}$} \\
\hline $\mathrm{O}_{\mathrm{F}}$ & $\begin{array}{l}\text { Environmental conditions where propagule production } \\
\text { is maximal }\end{array}$ & & \\
\hline$\sigma_{\mathrm{F}}$ & $\begin{array}{l}\text { Propagule production in sub-optimal conditions } \\
\text { (reproductive niche breadth) }\end{array}$ & & \\
\hline $\mathrm{m}_{\mathrm{W}}$ & Maximal probability of resisting extinction & \multirow{3}{*}{$W_{i x}=m_{W_{i}} \exp$} & \multirow{3}{*}{$\begin{array}{l}\text { Probability of local } \\
\text { extinction }\end{array}$} \\
\hline $\mathrm{o}_{\mathrm{W}}$ & $\begin{array}{l}\text { Disturbance level where resistance to extinction is } \\
\text { maximal }\end{array}$ & & \\
\hline$\sigma_{\mathrm{W}}$ & $\begin{array}{l}\text { Capacity to resist extinction (broad vs narrow tolerance } \\
\text { for disturbances) }\end{array}$ & & \\
\hline
\end{tabular}

(Eq. 3). The adjacency matrix indicates connections by dispersal (1) and their absence (0). The links are weighted by the distance between them according to the dispersal kernel given at Eq. 3. We simulate totally random, regular gradient (environmental conditions ranging from 1 to 100 for all simulations), auto-correlated (patchy) and homogeneous distribution of the environmental conditions. For the auto-correlated landscape, we use the same algorithm as for aggregation. The environmental conditions are randomly distributed among squares but are more similar within cells which results in aggregated or patchy distribution of environmental conditions.

\section{Equilibriums}

The model is solved at equilibrium by setting Eq. 1 to 0 for every patch and then focusing on the occupancy at each patch $x$. In the case of an isolated species with no competitors in the regional species pool we find:

$\hat{p}_{i x}=\frac{I_{i x}\left(1-\sum_{j=1}^{S=1} p_{j x}\right)}{W_{i x}}$

Here the basic result from metapopulation theory applies (Hanski 1999): for a species to persist regionally, the

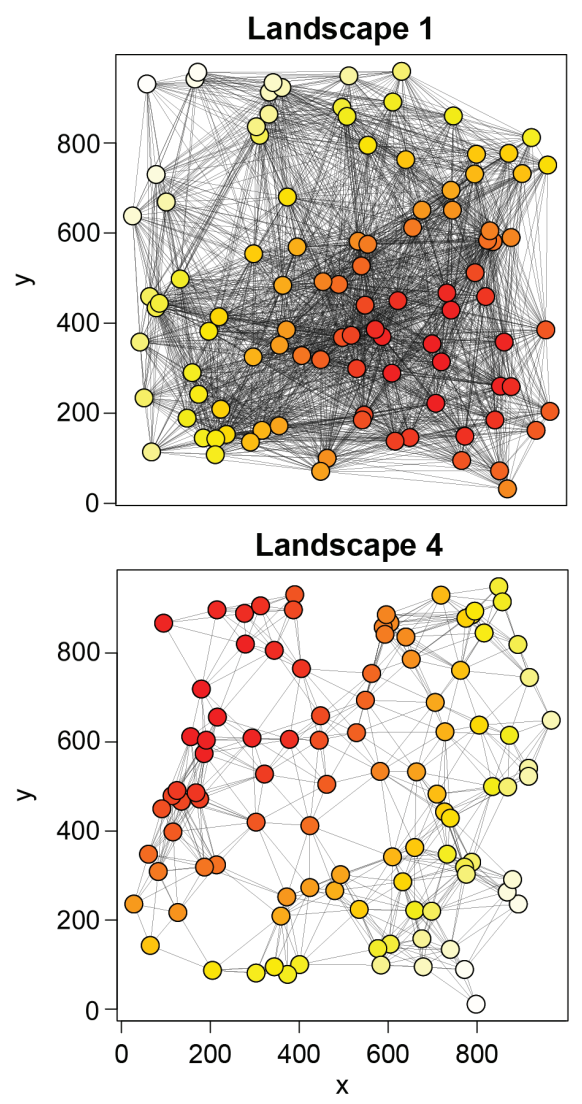

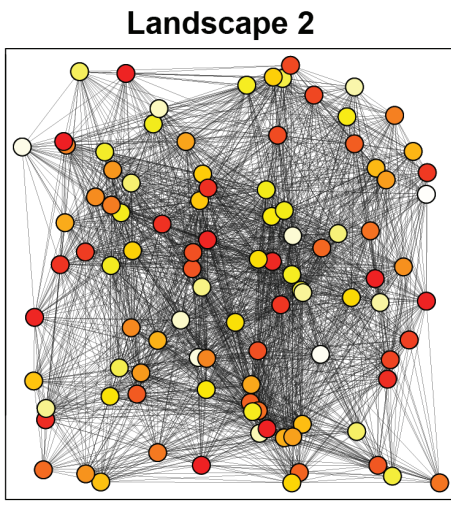

Landscape 5

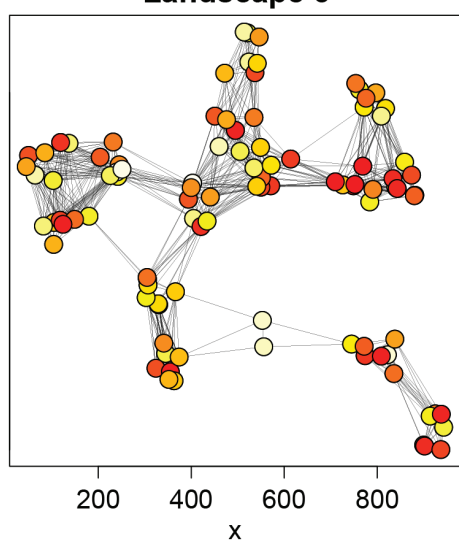

Landscape 3

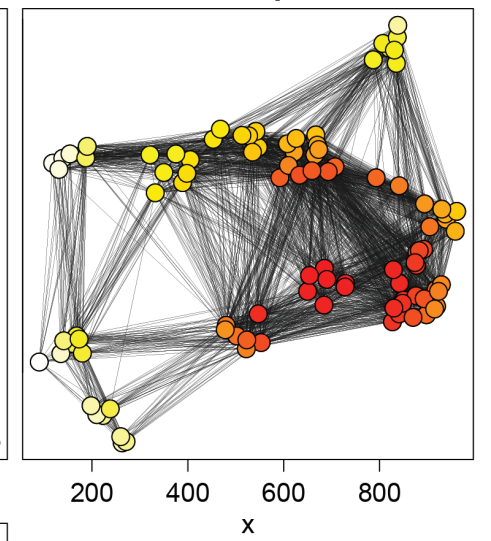

\begin{tabular}{cccc}
\hline Landscape & $\begin{array}{c}\text { Patch } \\
\text { connectivity }\end{array}$ & $\begin{array}{c}\text { Patch } \\
\text { agregation }\end{array}$ & $\begin{array}{c}\text { Environmental } \\
\text { heterogeneity }\end{array}$ \\
\hline 1 & High & Low & Gradient \\
2 & High & Low & Random \\
3 & High & High & Gradient \\
4 & Low & Low & Gradient \\
5 & Low & High & Random \\
\hline
\end{tabular}

Figure 2. Examples of landscapes with different patch aggregation, connectivity and distribution of environmental conditions. 
colonization probability must be larger than the extinction probability. The spatial heterogeneity in dispersal and environmental conditions however makes this equation tedious to solve and to relate to the average and the variance of landscape attributes. It is nonetheless easy to solve the model numerically and find the expected occupancy for each location in a given landscape. As previous metapopulation models have shown, we find that occupancy increases with the connection to the metapopulation, the area and the suitability of the patch (Moilanen and Hanski 1998).

The model can also be solved in presence of other species. When the displacement probability is null and there is perfect pre-emption (i.e. $\gamma=0$ ), then the probability that a species is present at a patch at equilibrium is given by:

$$
\hat{p}_{i x}=\frac{I_{i x}\left(1-R_{i x}-\sum_{j=1}^{S} p_{j x}\right)}{W_{i x}}
$$

For a species to persist regionally, the probability of colonizing an empty patch has to be higher than disturbanceinduced mortality in occupied ones. Here, the probability of colonizing an empty patch depends on both local conditions and the capacity of the competitors to colonize the same patch (including both the effects of propagule production and competitive ability).

The probability that a species is present at a patch at equilibrium when competitive displacement is allowed $(\gamma>0)$ is:

$$
\hat{p}_{i x}=\frac{I_{i x} \gamma R_{i x} \sum_{j \in C_{i x}>C_{j x}} p_{j x}+I_{i x}\left(1-R_{i x}-\sum_{j=1}^{S} p_{j x}\right)}{\gamma R_{j x}+W_{i x}}
$$

For a species to persist in the presence of another competitor, the probability of colonizing empty patches and that of excluding the competing species from occupied patches has to be higher than extinctions and the probability of being excluded by stronger competitors.

\section{Modeling the different coexistence mechanisms}

Equation 1 can be simplified in order to represent the different coexistence mechanisms: neutral dynamics, competition-colonization trade-offs, species sorting and mass effects (respectively N, CC, SS and ME). To do so, we can specify alternate parameterization of the full model to represent the assumptions of each mechanism (Supplementary material Appendix 1). The parameter settings for each case are summarized in Table 2. Hereafter, we refer to the model described in Eq. (1) as the full model when allowed to run without any parameter constraint; and as the sub-models when the parameters are forced to follow the assumptions of any one of the idealized coexistence mechanism.

\section{Evaluating the relative importance of the four coexistence mechanisms}

We compare the predictions of the full model to those of the four sub-models to partition the contribution of each mechanism to species distribution. We start from a pool of species with a known trait distribution. For the purpose of
Table 2. Summary of the parameter settings for each sub-model ( $\mathrm{N}$ neutral dynamic, $\mathrm{CC}=$ competition-colonization trade-off, $\mathrm{SS}=$ species sorting, $\mathrm{ME}=$ mass effects and $\mathrm{F}=$ full model). Param eters are either forced to have equal values among species (dark gray) or are allowed to differ (light gray). Competitive ability (c), propagule production (F) and resistance to extinction (W) are Gaussian functions defined by three parameters: $m, o$ and $\sigma$ (Table 1 ). Maximal performance $(m)$ ranges from 0 to 1 . Niche optima $(o)$ can take any value along a gradient in environmental conditions ranging from 1 to 100 . Tolerance to different environmental conditions or niche breadth $(\sigma)$ ranges from 0 to $\infty$ where a species performs equally well in all patches independently of local conditions.

\begin{tabular}{llllllllll}
\hline & $m_{C}$ & $o_{C}$ & $\sigma_{C}$ & $m_{F}$ & $o_{F}$ & $\sigma_{F}$ & $m_{W}$ & $o_{W}$ & $\sigma_{W}$ \\
\hline $\mathrm{N}$ & & & & & & & & & \\
$\mathrm{CC}$ & & & & & & & & & \\
$\mathrm{SS}$ & & & & & & & & & \\
$\mathrm{ME}$ & & & & & & & & & \\
Full & & & & & & & & & \\
\hline
\end{tabular}

the simulations, we randomly attribute trait values to species from a uniform distribution. For simplicity we also assume that extinction probability is constant across the entire landscape and equal among species $\left(W_{i x}=0.1\right)$. We run the full model using this pool of species to simulate species distributions without any constraints on the importance of different coexistence mechanisms. We redo the same for each sub-model to obtain species distributions where the importance of the different mechanisms is known. To do so, we adjust model equations and parameters to the assumptions of the respective metacommunity paradigm. For example, when a parameter is assumed to be identical among species such as maximal performances in species sorting (Table 2), we attribute to each species its value averaged over the pool. The comparison between the sub-models and the full model reveals the contribution of each mechanism to species distribution. We describe the different steps of the analysis in more details below.

The equilibrium probabilities of observing a species at a patch predicted by the four sub-models are likelihood functions that could be evaluated for distribution data. The likelihood of a presence in a given patch given a sub-model is $p_{i x, \text { sup }}$ and $1-p_{i x, \text { sup }}$ is the likelihood that it is absent. We compute the log-likelihood of observing each species in each patch for all four sub-models (patch scale). This metric gives us an estimate of the contribution of each patch to different coexistence mechanisms. We sum the log-likelihood over all species and patches to have a global assessment of the fit of the sub-models to the simulation data (metacommunity scale). The log-likelihood could also be summed for each species over all patches to have a species-specific evaluation of the different mechanisms to their distribution (species scale). This approach thus allows investigating the changes in the importance of the four coexistence mechanisms at the metacommunity level as well as those potentially occurring within metacommunities at the species level and at the patch level.

We compare the likelihood values to assess the relative importance of the four mechanisms. A small difference between the full and the sub-model indicates that the coexistence mechanism represented in the sub-model contributes significantly to species distribution, while a large difference indicates that it does not contribute much. These 
differences can be quantified using the 'weight of evidence' approach where the difference between the likelihood of the full model and that of each sub model is divided by the likelihood of the full model. The weight of evidence provides a scaled measure between 0 and 1 that is better suited for comparisons among studies than raw likelihood values.

\section{Simulations}

We run simulations to investigate the impact of landscape properties on two species coexistence, species richness and the likelihood of the four coexistence mechanisms at the landscape and species levels. We also run simulations with one species to assess the impact of species parameters and landscape properties on metapopulation persistence (Supplementary material Appendix 2, Fig. A1). We first investigate the effect of landscape properties on critical conditions for regional coexistence. We run 2-species simulations in 5 contrasted landscapes that vary in patch connectivity, aggregation and in the type of environmental heterogeneity (Fig. 2). Using the full model, we investigate the impact of differences in maximal performances and differences in optimal conditions on coexistence. Using the CC sub-model, we investigate the impact on coexistence of different trade-off strengths between competitive ability and propagule production.

We then investigate how regional species richness is influenced by landscape properties under different metacommunity paradigms in species rich metacommunities. We run simulations on the 5 landscapes presented in Fig. 2 using the main and the 4 sub-models. We used 100 metacommunities composed of 20 species. Species have a high resistance to extinction in all patches $\left(\mathrm{m}_{\mathbb{W}}=0.9 ; \sigma_{\mathbb{W}}=\infty\right)$. All other parameters are determined by random sampling from uniform distributions $(o=[0,100] ; m=[0,1]$ and $\sigma=[0,100])$.

Before using the likelihood statistic, we test its capacity to discriminate among the different coexistence mechanisms by doing simulations that cross sub-models and trait distributions. In species sorting and mass effect, species have different environmental optima $(o=(25,75))$ but otherwise equal maximal performances and niche breadth $(\mathrm{m}=0.7$; $\sigma=30)$ for the three traits. In competition-colonization trade-offs, species have equal environmental optima $(o=50)$ and niche breadths $(\sigma=50)$ but have opposed maximal productions of propagules and competitive abilities $\left(\mathrm{m}_{\mathrm{Cl}}=1\right.$ $\left.-\mathrm{m}_{\mathrm{F} 1}=\mathrm{m}_{\mathrm{F} 2}=1-\mathrm{m}_{\mathrm{C} 2}\right)$. In neutral dynamic, the species performs equally well in all patches $(\mathrm{m}=0.7 ; \sigma=\infty)$.

We finally investigate how the relative contribution of the four coexistence mechanisms to species distribution might vary with landscape topology, environmental heterogeneity and disturbances for a broad range of scenarios. We use 100 pools of 20 species where species have equal high resistance to disturbances $\left(\mathrm{m}_{\mathrm{W}}=0.9 ; \sigma_{\mathrm{W}}=\infty\right)$ but otherwise random parameters sampled from uniform distributions $(o=[0,100]$; $\mathrm{m}=[0,1]$ and $\sigma=[0,100])$. We run simulations on the five landscapes presented in Fig. 2 for each pool of species. For each run, we calculate the likelihood of each coexistence mechanism at the species level and compare these measures among simulated scenarios.
All patches are randomly occupied by one species at the beginning of the simulations and simulations run for time 1500 steps. Model implementation and subsequent numerical analyses were done with R-3.1.2 (R Development core team) using packages 'rootSolve' (Soetaert 2009).

Data available from the Dryad Digital Repository: $<$ http://dx.doi.org/10.5061/dryad.sn788> (Fournier et al. 2016).

\section{Results}

We find for two species simulations that the likelihood statistic performs well in discriminating the different coexistence mechanisms in all cases but two (Table 3). When the two species have the same traits (neutral), log-likelihood values are equal for the neutral and competition-colonization trade-off models. Similarly, likelihoods are equal for the mass effect and species sorting models when applied to the mass effects scenario. Two species simulations also reveal that coexistence at the regional scale is influenced by landscape properties (Fig. 3). Coexistence is enhanced by landscape connectivity (landscapes 1, 2 and 3 versus 4 and 5). However, changing the distribution of environmental conditions (landscape 1 versus 2 ) or increasing patch aggregation (landscape 1 versus 3) does not influence coexistence, even for species sorting. Interestingly, using a random spatial distribution of environmental conditions, increasing patch aggregation and decreasing the connectivity among patches (landscape 1, 2, 3 and 4 versus 5) reduce coexistence to a greater extent than any of these modifications alone, suggesting a strong synergy among landscape properties. These simulations also show that low niche overlap resulting from distinct environmental preferences $\left(\mathrm{o}_{\mathrm{i}} \neq \mathrm{O}_{\mathrm{j}}\right)$ promotes coexistence only in poorly connected landscapes (Fig. 3d-e). In the case of the competition-colonization sub-model (Fig. 3f-j), relatively weak trade-offs between species colonization and competitive abilities allow coexistence in landscape 1 . However, stronger trade-offs are required for coexistence to occur when connectivity is low (landscape 4) and even more so when modifying the three landscape properties (landscape 5).

Simulations with 20 species give similar general results as the two-species simulations. We find that low connectivity decreases regional species richness in the full and neutral models (Fig. 4, landscapes 4 and 5). Similarly, low connectivity, high aggregation and randomly distributed

Table 3. Likelihood statistics of the simulations with 2 species. Loglikelihood statistics are produced by the comparisons of results of the full and each sub-model (row) for each set of species parameters (column). Species parameters are defined a priori to match the assumptions of each metacommunity paradigm. A sub-model is expected to retrieve the best likelihood value (i.e. closest to 0 ) when run with its associated species parameters.

\begin{tabular}{lllcc}
\hline & \multicolumn{4}{c}{ Species parameters } \\
\cline { 2 - 5 } Sub-model & \multicolumn{1}{c}{$\mathrm{N}$} & $\mathrm{CC}$ & $\mathrm{SS}$ & $\mathrm{ME}$ \\
\hline $\mathrm{N}$ & $\mathbf{- 1 1 2 . 3}$ & -129.4 & -130.2 & -131.9 \\
$\mathrm{CC}$ & $\mathbf{- 1 1 2 . 3}$ & $\mathbf{- 1 1 4 . 1}$ & -126.6 & -130.7 \\
SS & -114.1 .8 & -124.3 & $\mathbf{- 1 0 0 . 8}$ & $\mathbf{- 1 2 2 . 3}$ \\
ME & -116.4 & -124.8 & -125.5 & $\mathbf{- 1 2 2 . 3}$ \\
\hline
\end{tabular}



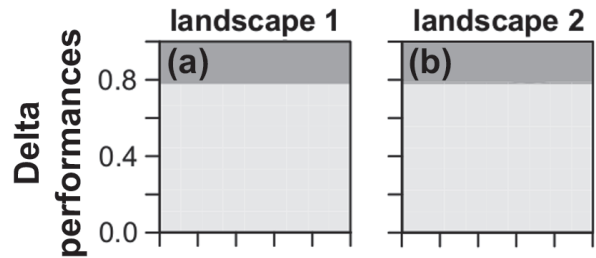

landscape 3
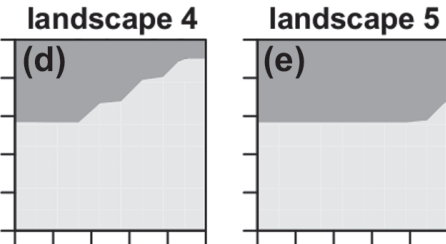

coexistence:

\section{Delta optima}
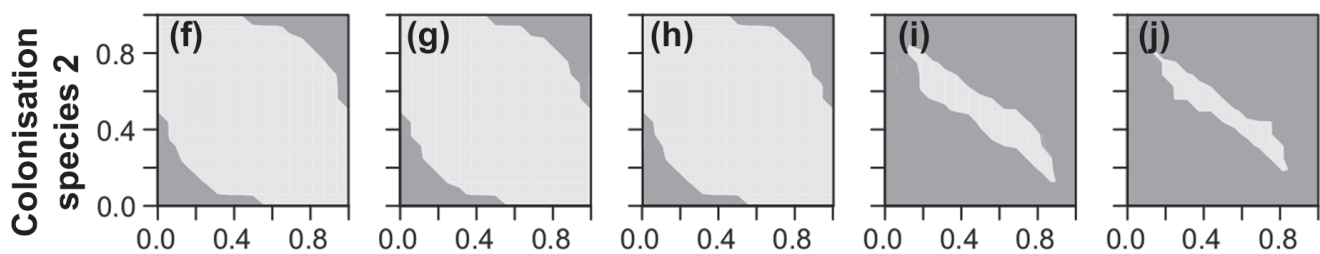

$=$ yes

$=$ no

\section{Colonisation species 1}

Figure 3. Impact of landscape properties on coexistence of 2 species using the full model $(\mathrm{a}-\mathrm{e})$ and the competition-colonization tradeoff sub-model $(f-j)$. The first row shows the probability of coexistence for different competitive abilities, with delta performances the absolute difference between species maximal performances $\left(\left|m_{i c}-m_{j c}\right|\right)$, and delta optima the absolute difference between species optimal environmental conditions $\left(\left|o_{i c}-o_{j c}\right|\right)$. Species with higher maximal performances are likely to exclude other species except when the differences in performances are too small or when difference between species optimal environmental conditions are too high leading to low interspecific competition. The second row uses the CC sub-model to show the changes in the probability of coexistence for different trade-offs between species competitive ability and propagule production (illustrated as a function of the propagule production of species 1 and 2).

environmental conditions decrease species richness under mass effect. However, landscape properties do not impact species richness under species sorting and competitioncolonization trade-off. In this respect, species sorting produces the highest species richness and competitioncolonization trade-off the lowest in all landscapes (Fig. 4).

We find that the likelihoods of the four coexistence mechanisms vary significantly among landscapes and among species (Fig. 5). The variation among landscapes is relatively weak, but some coexistence mechanisms are nonetheless more important than others in given conditions. For example, species distribution on landscape 3 better corresponds to the expectancy of species sorting and competitioncolonization trade-offs than that of neutral dynamic and mass effect. There is however more variation among species than landscapes in the importance of the four coexistence (a)

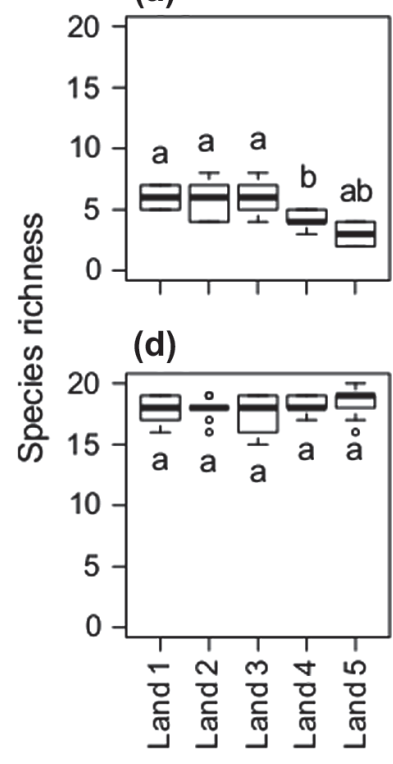

(b)

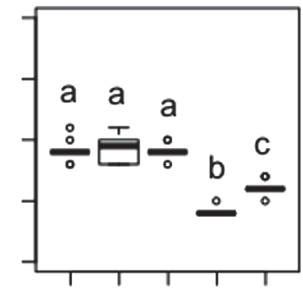

(e)

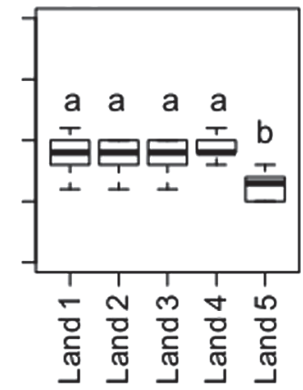

(c)

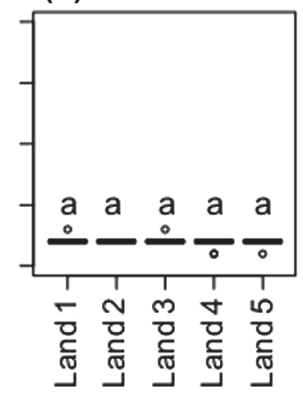

Figure 4. Effect of landscape configuration and metacommunity coexistence mechanisms on regional species richness. Simulations were run on the five landscapes presented in Fig. 1 using the full and the four sub-models $((a)=$ full model, $(b)=$ neutral dynamic, $(c)=$ competition-colonization trade-off, $(\mathrm{d})=$ species sorting and $(\mathrm{e})=$ mass effects). Each simulation is repeated 100 times with a different pool of species representing a total of 2500 simulations. Each pool is constituted by 20 species with random trait values. Letters indicate significant changes among landscapes (Tukey honest significant differences). 


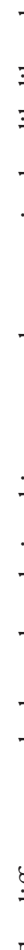

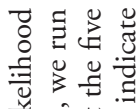

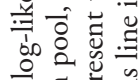

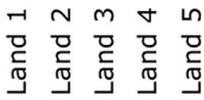

戈空至

岛

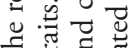

oo

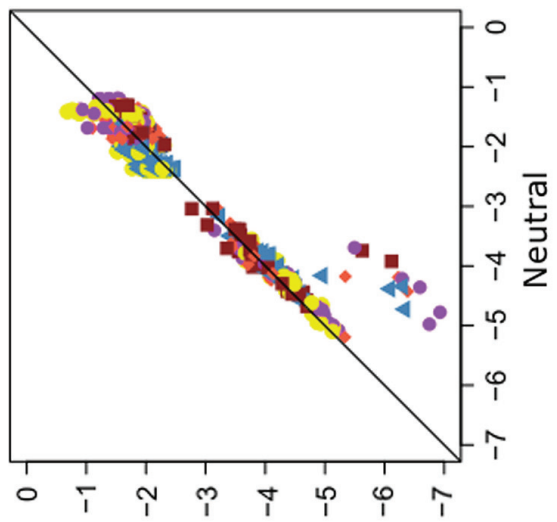

дวәرд ssew

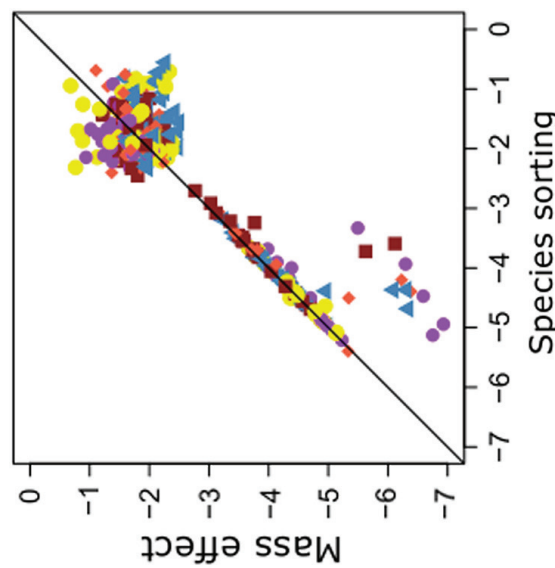

है है

की

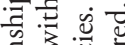

os

政

का

ક워

3

s.

ฐี

范

乐苞总

E位的

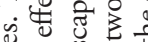

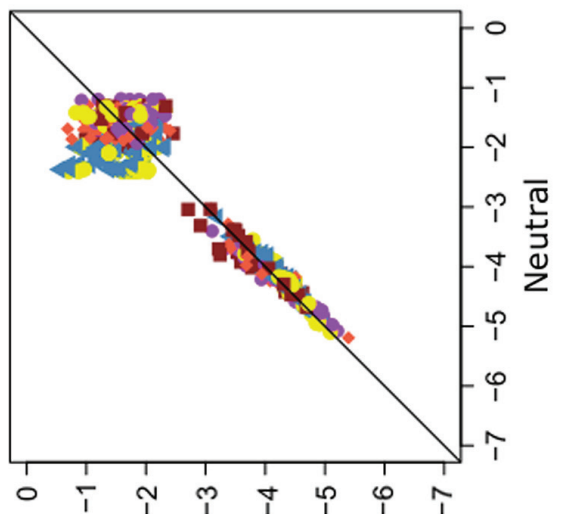

bu!nuos sə!̣วds
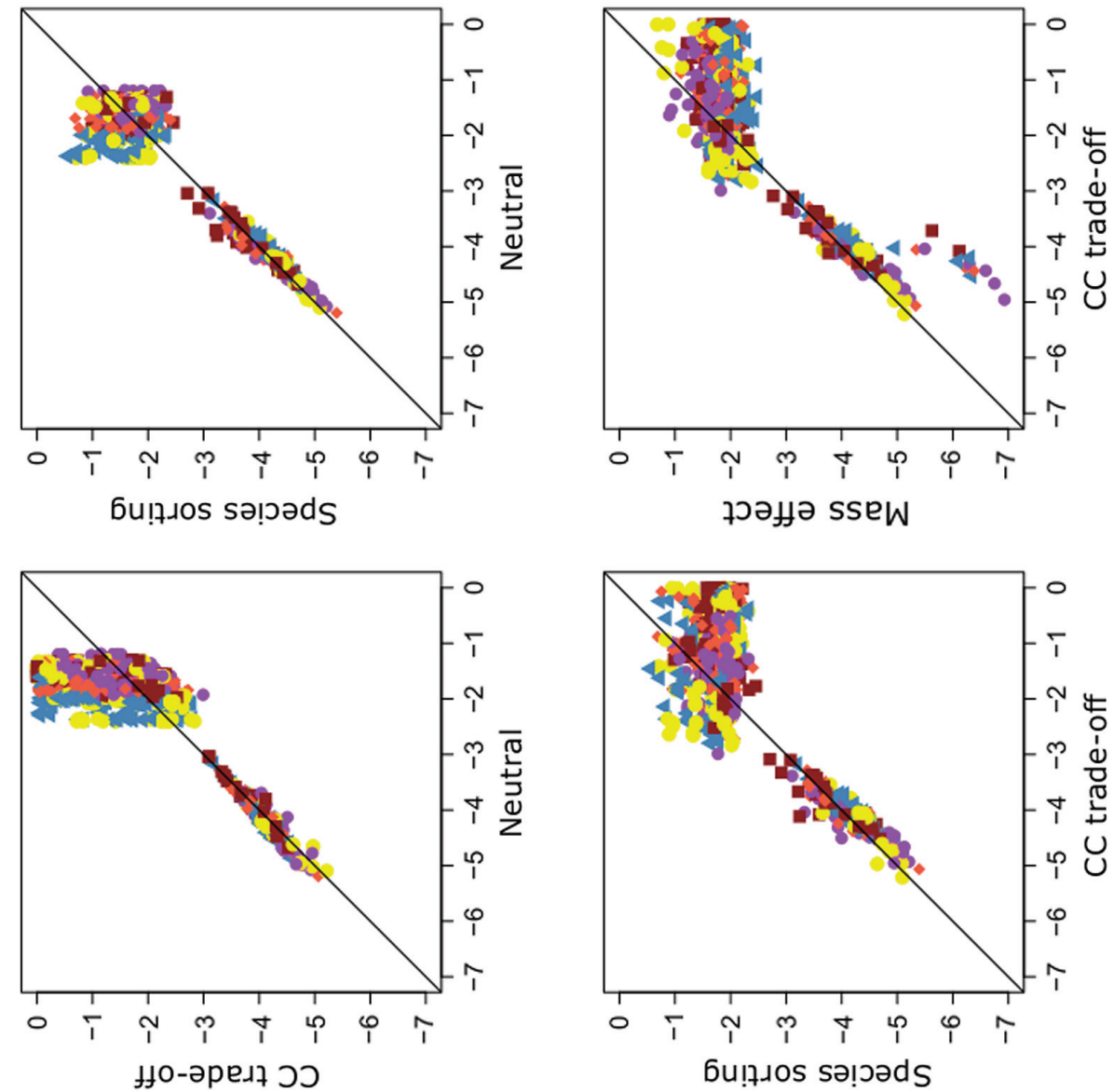

设

可式合索

पू तु

点.

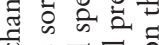

पु.

递总密

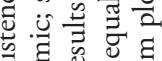

政

웡 흥

둔

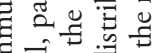

중 3

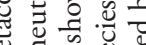

品

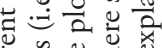

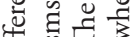

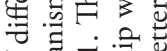

प등-寻

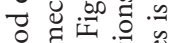

品.

च्य च च

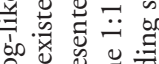

잉

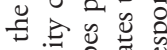

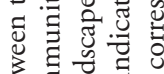

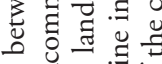

茟苋

表范菏

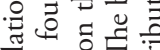

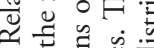

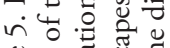

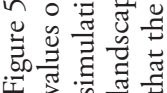


mechanisms (Fig. 5). We observe, within each landscape, substantial variation among species in the relative importance of the four mechanisms.

\section{Discussion}

Leibold et al. (2004) summarized four coexistence mechanisms operating at the metacommunity scale. These are often oversimplified as alternative, mutually exclusive hypotheses. In reality, they arise from various combinations of drift, dispersal, niche differentiation and competitive hierarchies (Mouquet et al. 2005, Vellend 2010, Livingston et al. 2012). Since then, a number of studies have tried to assess which of them best explains the observed distribution of species (Cottenie 2005, Pandit et al. 2009, Livingston et al. 2013, Yeh et al. 2014). The last decade has also seen an increasing effort to move from opposing the four coexistence mechanisms toward their integration into a common framework (Gravel et al. 2006, Leibold and McPeek 2006, Adler et al. 2007, Chase and Myers 2011). The present study makes a further step toward the development of a general theory of metacommunity ecology. The modeling framework provides 1) a formal mathematical integration of the four coexistence mechanisms, and also a wide range of intermediate situations, 2) the capacity to compute their likelihood in various ecological and spatial contexts and 3) the capacity to model landscape properties and the distribution of species, traits and environmental conditions. Our approach contrasts with previous models that considered fewer and/or non-simultaneous mechanisms (Münkemüller et al. 2012, Ai et al. 2013, Matias et al. 2014).

Landscape and species characteristics influence the relative contribution of the different mechanisms and the number and type of species that persist. Dispersal limitations due to low patch connectivity emerge as a key factor for metacommunity organization (Moritz et al. 2013). In absence of environmental constraints, we find that connectivity is the only factor impacting patch occupancy by a single species metapopulation, with a threshold connectivity required for persistence (Supplementary material Appendix 2, Fig. A1). Below this threshold, a landscape consists of several subnetworks among which dispersal does not occur. This result recalls the importance of fragmentation for metapopulation persistence (Hanski 1998). The parameter space where coexistence is possible is also more constrained and regional species richness lower in landscapes with low patch connectivity. In other words, dispersal limitation makes it more difficult for two or more species to coexist. Gravel et al. (2011) found similar results when investigating the importance of connectivity for persistence in trophic metacommunities. They found that connectivity promotes coexistence at the regional scale by reducing the negative impact of dispersal limitations and by increasing the access to key resources. Similar effects are likely to operate in our metacommunities with high connectivity making it easier for a species to reach a patch where environmental conditions are optimal.

We find that interactions among landscape properties occur and can constrain coexistence to a greater extent than connectivity alone. For instance, changing patch aggregation, connectivity and the distribution of environmental conditions constrains the conditions for coexistence to a greater extent than changing any of these landscape properties alone. Due to these interactions, we find the highest regional species richness with species-sorting and no dispersal limitation and the lowest richness with neutral or mass effect and dispersal limitation. The importance of dispersal limitation in the neutral or mass effect sub-models is further highlighted by the lower species richness observed in landscapes with low connectivity of patches. In the competition-colonization sub-model, the best regional competitors and colonizers exclude the other species thus explaining the low richness obtained. In the case of the full model, the best local competitors and colonizers exclude other species from patches within a given range of environmental conditions, thus explaining the intermediate richness.

Our results show that not only the importance of the coexistence mechanisms can vary among landscapes, it also varies among species. Some species in a landscape could have distributions that are strongly limited by dispersal limitations, while other species have distributions that are strongly related to environmental conditions. This result confirms that the importance of coexistence mechanisms depends on the scale at which they are investigated (Chase 2014) and calls for considering scales as continuous within and among regions instead of the currently compartmentalized perception that dominates in community and metacommunity ecology (Ricklefs 2008). Moreover, it suggests that the success of biodiversity management is not only site-specific but also species-specific. It is common sense to find that a management strategy can be successful for some species but not for others. And, species responses to a given management strategy might differ among locations because the importance of dispersal and environmental conditions varies. For example, restoring lateral connectivity in a floodplain along the Rhone River favored alien macroinvertebrates better adapted to flow (Paillex et al. 2015). However, this result depends on the species pool present at the surroundings of the study site (Sundermann et al. 2011).

Dispersal limitation, although not adequately considered in the original set of four coexistence mechanisms at play in a metacommunity (Leibold et al. 2004), emerge as a key process in our framework. Dispersal limitation is somehow canonical to the mass effect, as they can lead to the replacement of superior competitor by inferior ones (Hurtt and Pacala 1995). The mechanisms responsible for this disconnect between competitivity and relative abundance in the pool of propagules differ however, as one is caused by overabundance of weak competitors (the mass effect) and the other by recruitment failures of the top competitor (dispersal limitations). Dispersal limitations act as an equalizing mechanism of coexistence (Chesson 2000), meaning that they could promote coexistence at the regional scale only if other stabilizing mechanisms, such as niche differentiation, are already at play. It is nonetheless a particularly important one because dispersal is often a limiting process in natural communities, promoting local diversity by slowing down competitive exclusion. Dispersal limitation influences both the distribution of diversity (Cadotte 2006, Myers and Harms 2009, Condit et al. 2012) and the nature and strengths of species interactions (Shurin and Allen 2001, Chase et al. 2010). The mass effect results in similar species distribution 
to dispersal limitation, with species not systematically found where they are the most competitive. However, the mass effect requires a fine environmental grain as compared to species dispersal capacity (Mouquet and Loreau 2002), while dispersal limitation can happen on any landscape. The conditions for the mass effect to happen are constraining and more likely to be met at small spatial scales. Mass effect is thus likely to be much less important for species distribution at large scales than dispersal limitation. This was problematic in the original metacommunity framework because dispersal limitation were not considered as a mechanism on its own. Our framework (i.e. the full model and the four sub models, the likelihood method and the associated species traits and landscape configuration) solves this issue as it allows investigating the likelihood of mass effect and dispersal limitations with a mechanistic derivation of the expected distribution, instead of the passive description of the observed distribution obtained with variance partitioning methods.

We expect the model to be general enough to be eventually applicable to empirical data. Hanski and collaborators pioneered the parameterization of the incidence function model to empirical data with model organisms such as the Glanville fritillary, the Americane pika and the European nuthatch (see the review in Hanski 1999). The model we propose is complicated by the ecological interactions taking place between species, but it nonetheless builds on the same template as Hanski did with the traditional Levins metapopulation model. The occurrence of a species in a given locality is represented statistically as a binomial process conditional on the patch connectivity, its area and environmental characteristics (Moilanen and Hanski 1998). The occurrence of a species could therefore be modeled with the routine of a generalized linear model, or alternatively a more flexible algorithm if non-linearities are important. It is easy to interpret the terms of the linear model as colonization and extinction processes and ecological interactions are introduced as modifiers of these (Gravel et al. 2011). Leroux et al. (pers. comm.) recently applied the approach to investigate the impacts of landscape fragmentation on biodiversity distribution. They found that, in support of our investigation here, the combination of patch dynamics and species sorting best explained the distribution of several emblematic species of temperate forests in eastern Canada. Different approaches to spatial dynamics were found complementary to each other, highlighting the structural uncertainty arising from different model structures. At the end, the most accurate and reliable model was found to be the integrated one with the different dynamics at play simultaneously.

The likelihood functions can be estimated directly from spatially explicit presence/absence data. The probability of presence of a species in a local community can be estimated with the different sub-models. In our idealized scenarios, we already have information about species traits, making the evaluation of the incidence functions straightforward. In reality, information about species performances along an environmental gradient are required. Here, species distribution data, species traits or other relevant biological variables related to competitive ability, propagule production or extinction resistance can be used to estimate $C_{i x}, F_{i x}$ and $W_{i x}$ respectively. Niche optima, ranges and maximal performances are derived from $C_{i x}, F_{i x}$ and $W_{i x}$. In addition, information about species dispersal capacity are required to have a realistic representation of spatial processes at the landscape scale. Parameters that are difficult to estimate from the available data can be set by hypothesis. For example, plant competitive ability for light $\left(C_{i x}\right)$ can be estimated through local measurement of specific leaf area. The number of seeds provides an estimation of plant propagule production $\left(F_{i x}\right)$. Plant survival to natural disturbance such as floods or fire provide an estimation of the extinction probability in disturbed environments $\left(W_{i x}\right)$. Plant dispersal capacity can be estimated using seed size or it can be derived from species distribution maps. Boulangeat et al. (2012) performed a similar analysis, showing that the addition of dispersal limitation and a proxy of biotic interactions improve significantly the performance of species distribution models. While their statistical approach is less constrained than ours, they used a similar formulation of dispersal limitation and found that it better explains why species could be absent from locations they perform best (dispersal limitations), and present in sink locations (mass effect). See Supplementary material Appendix 3 for further guidelines on model application.

Applying the framework to real data constitutes a mechanistic hypothesis-driven alternative aproach to the phenomenological approach of variance partitioning (Cottenie 2005). The likelihood of each coexistence mechanism could be evaluated and the models compared by their relative weight of evidence. Fitted parameters could also be studied directly. For simplicity, we assumed Gaussian distribution of species performance along environmental gradients and equal dispersal among species. These assumptions should be verified and, when necessary, relaxed before applications to real data. The existing methods to relate field data to coexistence mechanisms are based on the species and/or trait distribution. For example, diversity indices (Mason et al. 2012, Münkemüller et al. 2012, Spasojevic and Suding 2012) and variance partitioning among environmental and spatial components (Cottenie 2005) are used to assess their relative importance. Our framework overcomes some caveats of these approaches as 1) it allows explicit tests of the importance of dispersal limitations and 2) it considers the different coexistence mechanisms jointly in a common framework. The framework thus constitutes a complementary tool to investigate the influence of spatial geographic structure on coexistence and their associated biodiversity distribution.

While the model performs well at the landscape scale, it does not consider coexistence within local communities. Indeed, patches host a population of single species in our model. This assumption is unrealistic but a different interpretation of the model could overcome this limitation. Patches can be interpreted as micro-sites hosting a single individual (see for instance Tilman et al. 1994). Under this scenario, local communities can be defined as a group of neighbor micro-sites. It follows that the scale from local to global (landscape scale) can be made continuous by progressively increasing the number of micro-sites included in a local community. This offers the possibility to adapt the likelihood statistic to assess how the importance of the four metacommunity paradigms changes across scales (Chase 2014). Such an approach remains to be developed and could provide interesting insights on the importance of scale for ecological processes. 
Integrating the four coexistence mechanisms of metacommunity theory and dispersal limitations into a common spatially explicit modeling framework provides important insight about spatial processes in metacommunities. Our results particularly highlight the importance of dispersal limitation as a driver of species distribution and the strong synergies between landscape properties and coexistence mechanisms. Moreover, they strongly suggest that natural communities may often be the result of multiple mechanisms acting simultaneously at different scales. This complexity has to be considered in species distribution models in order to improve our capacity to predict future changes in biodiversity. Future studies should seek ways to better integrate coexistence mechanisms including speciation that was not considered here and spatial processes in species distribution.

Acknowledgements - We thank Isabelle Gounand, Isabelle Boulangeat and Jonathan Chase for helpful advises. Financial support was provided to $\mathrm{BF}$ by the Swiss National Science Foundation (SNSF), projects P2NEP3_148841. NM was supported by the CNRS. MAL was supported by NSF DEB-1353919. DG was supported by the NSERC and the Canada Research Chair program.

\section{References}

Adler, P. B. et al. 2007. A niche for neutrality. - Ecol. Lett. 10: 95-104.

Ai, D. et al. 2013. Spatial structures of the environment and of dispersal impact species distribution in competitive metacommunities. - PLoS One 8: e68927.

Amarasekare, P. et al. 2004. Mechanisms of coexistence in competitive metacommunities. - Am. Nat. 164: 310-326.

Biswas, S. and Wagner, H. 2012. Landscape contrast: a solution to hidden assumptions in the metacommunity concept? - Landscape Ecol. 27: 621-631.

Boulangeat, I. et al. 2012. Accounting for dispersal and biotic interactions to disentangle the drivers of species distributions and their abundances. - Ecol. Lett. 15: 584-593.

Cadotte, M. W. 2006. Dispersal and species diversity: a metaanalysis. - Am. Nat. 167: 913-924.

Calcagno, V. et al. 2006. Coexistence in a metacommunity: the competition-colonization trade-off is not dead. - Ecol. Lett. 9: 897-907.

Carrara, F. et al. 2012. Dendritic connectivity controls biodiversity patterns in experimental metacommunities. - Proc. Natl Acad. Sci. USA 109: 5761-5766.

Chase, J. M. 2014. Spatial scale resolves the niche versus neutral theory debate. - J. Veg. Sci. 25: 319-322.

Chase, J. M. and Myers, J. A. 2011. Disentangling the importance of ecological niches from stochastic processes across scales. - Phil. Trans. R. Soc. B 366: 2351-2363.

Chase, J. M. et al. 2010. Habitat isolation moderates the strength of top-down control in experimental pond food webs. - Ecology 91: 637-643.

Chesson, P. 2000. Mechanisms of maintenance of species diversity. - Annu. Rev. Ecol. Syst. 31: 343-366.

Condit, R. et al. 2012. Thirty years of forest census at Barro Colorado and the importance of immigration in maintaining diversity. - PLoS One 7: e49826.

Cottenie, K. 2005. Integrating environmental and spatial processes in ecological community dynamics. - Ecol. Lett. 8: 1175-1182.

Economo, E. P. and Keitt, T. H. 2008. Species diversity in neutral metacommunities: a network approach. - Ecol. Lett. 11: 52-62.
Economo, E. P. and Keitt, T. H. 2010. Network isolation and local diversity in neutral metacommunities. - Oikos 119: 1355-1363.

Fournier, B. et al. 2016. Data from: An integrative framework of coexistence mechanisms in competitive metacommunities. - Dryad Digital Repository, <http://dx.doi.org/10.5061/ dryad.sn788>

Gravel, D. et al. 2006. Reconciling niche and neutrality: the continuum hypothesis. - Ecol. Lett. 9: 399-409.

Gravel, D. et al. 2011. Trophic theory of island biogeography. - Ecol. Lett. 14: 1010-1016.

Gravel, D. et al. 2014. Using neutral theory to reveal the contribution of meta-community processes to assembly in complex landscapes. - J. Limnol. 73: 61-73.

Hanski, I. 1994. A practical model of metapopulation dynamics. - J. Anim. Ecol. 63: 151-162.

Hanski, I. 1998. Metapopulation dynamics. - Nature 396: 41-49.

Hanski, I. A. 1999. Metapopulation ecology. - Oxford Univ. Press.

Hubbell, S. P. 2001. The unified neutral theory of biodiversity and biogeography. - Princeton Univ. Press.

Hubbell, S. P. et al. 1999. Light-gap disturbances, recruitment limitation, and tree diversity in a neotropical forest. - Science 283: 554-557.

Hurtt, G. C. and Pacala, S. W. 1995. The consequences of recruitment limitation: reconciling chance, history and competitive differences between plants. - J. Theor. Biol. 176: 1-12.

Leibold, M. A. 2011. The metacommunity concept and its theoretical underpinnings. - In: Scheiner, S. and Willigg, M. (eds), The theory of ecology. Univ. of Chicago Press, pp. 163-184.

Leibold, M. A. and McPeek, M. A. 2006. Coexistence of the niche and neutral perspectives in community ecology. - Ecology 87: 1399-1410.

Leibold, M. A. et al. 2004. The metacommunity concept: a framework for multi-scale community ecology. - Ecol. Lett. 7: 601-613.

Levins, R. 1969. Some demographic and genetic consequences of environmental heterogeneity for biological control. - Bull. ESA 15: 237-240.

Levins, R. and Culver, D. 1971. Regional coexistence of species and competition between rare species. - Proc. Natl Acad. Sci. USA 68: 1246-1248.

Livingston, G. et al. 2012. Competition-colonization dynamics in experimental bacterial metacommunities. - Nat. Commun. 3: 1234.

Livingston, G. et al. 2013. Do species sorting and mass effects drive assembly in tropical agroecological landscape mosaics? - Biotropica 45: 10-17.

Logue, J. B. et al. 2011. Empirical approaches to metacommunities: a review and comparison with theory. - Trends Ecol. Evol. 26: 482-491.

Mason, N. W. H. et al. 2012. A guide for using functional diversity indices to reveal changes in assembly processes along ecological gradients. - J. Veg. Sci. 24: 794-806.

Matias, M. G. et al. 2014. Estimates of species extinctions from species-area relationships strongly depend on ecological context. - Ecography 37: 431-442.

Moilanen, A. and Hanski, I. 1998. Metapopulation dynamics: effects of habitat quality and landscape structure. - Ecology 79: 2503-2515.

Moritz, C. et al. 2013. Disentangling the role of connectivity, environmental filtering, and spatial structure on metacommunity dynamics. - Oikos 122: 1401-1410.

Mouquet, N. and Loreau, M. 2002. Coexistence in metacommunities: the regional similarity hypothesis. - Am. Nat. 159: 420-426.

Mouquet, N. et al. 2005. The world is patchy and heterogeneous! Trade-off and source-sink dynamics in competitive 
metacommunities. - In: Holyoak, M. et al. (eds), Metacommunities: spatial dynamics and ecological communities. Univ. of Chicago Press, pp. 237-262.

Münkemüller, T. et al. 2012. From diversity indices to community assembly processes: a test with simulated data. - Ecography 35 : 468-480.

Myers, J. A. and Harms, K. E. 2009. Local immigration, competition from dominant guilds, and the ecological assembly of high-diversity pine savannas. - Ecology 90: 2745-2754.

Paillex, A. et al. 2015. Testing predictions of changes in alien and native macroinvertebrate communities and their interaction after the restoration of a large river floodplain (French Rhône). - Freshwater Biol. 60: 1162-1175.

Pandit, S. N. et al. 2009. Contrasts between habitat generalists and specialists: an empirical extension to the basic metacommunity framework. - Ecology 90: 2253-2262.

Ricklefs, R. E. 2008. Disintegration of the ecological community. - Am. Nat. 172: 741-750.

Shurin, J. B. and Allen, E. G. 2001. Effects of competition, predation, and dispersal on species richness at local and regional scales. - Am. Nat. 158: 624-637.

Supplementary material (Appendix ECOG-02137 at < www. ecography.org/appendix/ecog-02137>). Appendix 1-3.
Soetaert, K. 2009. rootSolve: nonlinear root finding, equilibrium and steady-state analysis of ordinary differential equations. - R-package ver. 1.6.

Spasojevic, M. J. and Suding, K. N. 2012. Inferring community assembly mechanisms from functional diversity patterns: the importance of multiple assembly processes. - J. Ecol. 100: 652-661.

Sundermann, A. et al. 2011. River restoration success depends on the species pool of the immediate surroundings. - Ecol. Appl. 21: 1962-1971.

Tilman, D. 1994. Competition and biodiversity in spatially structured habitats. - Ecology 75: 2-16.

Tilman, D. et al. 1994. Habitat destruction and the extinction debt. - Nature 371: 65-66.

Urban, D. L. et al. 2009. Graph models of habitat mosaics. - Ecol. Lett. 12: 260-273.

Vellend, M. 2010. Conceptual synthesis in community ecology. - Q. Rev. Biol. 85: 183-206.

Yeh, Y. C. et al. 2014. Determinism of bacterial metacommunity dynamics in the southern East China Sea varies depending on hydrography. - Ecography 38: 198-212. 